\title{
Effect of Yarn Characteristics on the Needle Contamination during Knitting Process
}

\author{
Young-Seok Koo \\ Department of Clothing \& Textiles, Pusan National University, Busan, South Korea 609-735
}

(Received: February 3, 2010/Revised: March 17, 2010/Accepted: March 22, 2010)

\begin{abstract}
Needle contamination was investigated using a test rig simulated the knitting points in the knitting machine in relation with the characteristics of knitting yarn such as yarn type and moisture contents. When used open-end yarn compared with combed and carded ring-spun yarns, the needle contamination was significantly increased because of the higher friction between yarn and needle by the fly and other impurities. Also Lower moisture content of the yarns aggravated the needle contamination. In addition knitting yarn breakage was substantially influenced by the higher needle contamination.
\end{abstract}

Keywords: needle, contamination, fly, yarn type, yarn characteristics

\section{Introduction}

Needle contamination is a polluted problem by a short fiber and tiny substances at the knitting points in the knitting machine. Specially, fly (lint) is a loose fiber that broken from the yarn composition during the knitting processing. As it is relatively small and lightweight it is suspended in the atmosphere, contacts with an obstacle and comes to rest ${ }^{1,2)}$. The fly problem is mainly caused by the friction between yarns and knitting elements on the knitting machine. Particularly, lint generation in the knitting zone in the knitting machine is critical to decide the quality of knitted products and knitting process. The tiny substances such as dust and wax derived from the base yarns such as cotton make the problem even worse in the knitting process ${ }^{3)}$.

In the process of knitting, yarn is converted into knitted loops at the knitting points in the knitting machine. In order to achieve this, it is necessary to deliver the yarn with great precision to the latch needles by means of yarn feeders.

The knitting points represents a typical example of fly being created because of extreme yarn diversions, i.e. narrow radii points of friction and drastically increased yarn stresses produce an ideal condition for the accumulation of fly and other impurities ${ }^{4}$.

During the stitch forming cycle in the knitting process, the yarn is subjected to high tension, frictional forces and bending stresses between yarns and knitting elements ${ }^{5}$. On the knitting machines, the yarn is delivered to the yarn guides readily after the yarn guides deliver yarn tangentially to the needles. This sudden change of yarn feeding angle results in a load to the yarn which causes fly shedding.

Besides the yarn is forced to bend to a great degree by the tiny radius of the hook of the needles, and the stress between the yarn and the

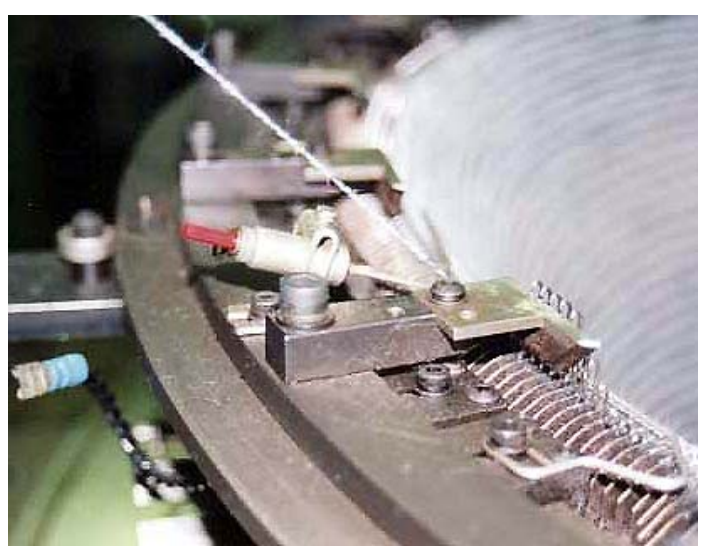

Fig. 1. Knitting points in a knitting machine.

${ }^{\dagger}$ Corresponding author. Tel.: +82-51-510-2843; Fax.: +82-51-583-5975; e-mail: youngskoo@pusan.ac.kr 
knitting elements causes the yarn to deform in cross section. This, in turn, results in a momentary localized twist change in the yarn. This may well increase the frictional forces between the yarn and the knitting elements and between fibers themselves, substantially contributing to fiber fly generation $^{6,7)}$.

Specifically, the greatest amount of fly at the knitting point occurs during the interlooping of yarns when the needles are in their lowest position.

Serious problems caused by the generation of fly are the decrease in working efficiency of the machine and defects on knitted fabrics. These faults in knitted fabrics, especially when cotton yarns are knitted on high production circular knitting machine, are frequently due to fly. Many fault analysis projects have shown conclusively that $60-80 \%$ of faults are caused by fly. Thus the costs incurred by these faults may be up to $4-6 \%$ of the total turnover $^{8)}$.

The fly can also cause quality, health, fire as well as maintenance of knitting machine problems. The fact that it accounts for $15 \%$ of all faults, $15 \%$ of downtime for maintenance, and weight losses between 0.5 and $1 \%$ of a knitted fabric demonstrates the importance of the fly problem. Therefore, the important fact is that the fly problem influences all aspects of knitting process ${ }^{9}$.

One of the reasons to the needle contamination is a yarn type. Spun yarns are produced from natural or synthetic fibres.

Under certain conditions, fly can occur when using either of these yarn types. Some synthetic fibers contain matting agents such as titanium dioxide which deteriorated the fly problem. Natural fibres containing foreign particles exercise a similar effect. Depending on where to be cultivated, harvesting method, weather conditions and ginning, natural fibers can contain varying degrees of impurity ${ }^{10)}$.

Some cottons are contaminated by sand dust which is not eliminated by the preparations for spinning and during the spinning process itself, inevitably causing not only fly generation but also wearing of needle elements. Another cause of the fly generation is a spinning process of yarn. Open-end spinning has high productivity and lower manufacturing costs due to high spinning speed and fewer work stages, while ring spinning has lower productivity and higher manufacturing costs due to lower spinning speed and greater number of work stages.

In the respect of yarn structure, they have different physical characteristics. Untidy open-end spun yarn in lengthways and crossways has fiber pattern with wrapping fibers which induce greater transverse vibration of the needle, an unsteady needle action, greater friction on the needle, greater contact pressure of the yarn and the stitch on the needle. On the other hand, ring spun yarn has a smooth and parallel pattern, less marked transverse vibration acting on the needle, smoother needle action, lower friction on the needle, and lower contact pressure of the yarn and the stitch on the needle. The differentiated characteristics of the yarn structure largely affect knitting faults such as fly generation and needle wearing during the knitting process ${ }^{11)}$.

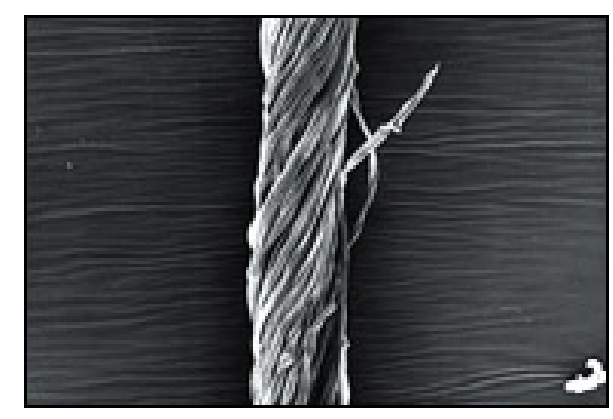

Ring-spun yarn

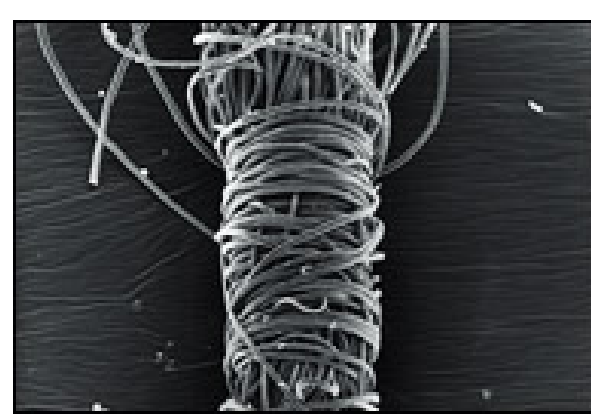

Open-end yarn

Fig. 2. Yarn structure. 
Another concern of the yarn structure relative to the needle contamination is yarn impurity. Open-end yarn contains a large number of mineral and vegetable impurities in the structure of the yarn which act on the stitch forming elements. However, ring spun yarn contains only few mineral or vegetable particles deposited on the yarn structure.

In the case of cotton yarn manufacturing, depending on the time and effort invested in the preparations for spinning, it is possible to considerably improve the purity regarding abrasion inducing particles. Certainly the open end method permits the reduction of processes and the use of shorter and higher contaminated cotton, with the result that these yarns create a higher friction with needles and then needle contamination. This abrasive effect can be minimized by a careful selection of raw materials, and intensive purification and dust removal during preparation and process for spinning ${ }^{12}$. The purpose of this study is to investigate the degree of needle contamination during knitting process, especially, in the knitting area. The knitting zone in the knitting machine is a critical area to decide the quality of knitted product.

However, the knitting area is readily contaminated by the frictional force between needles and yarn, which makes the process slow for cleaning the knitting zone. In addition, as the knitting zone is very complicated part in the machine, it is hard to examine the evaluation of the contamination. Therefore, this study focuses on the extent of needle contamination with different yarn type and moisture content of yarn by a developed test rig which simulates the knitting zone in the machine.

\section{Experimental}

The test-rig was designed to simulate the knitting points on the knitting machine and it was also specifically designed to measure the amount of impurities on the inside of needle path. The test-rig consists of an enclosed area, in which a knitting needle(28G, latch needle) is mounted on a stand, yarn feeding rollers, tension measuring sensors and yarn guides. Different gauges of needles can be mounted on the stand in the enclosed area.

In order to monitor the yarn tension variation, two tension measuring heads, which have a maximum frequency of $200 \mathrm{~Hz}$, a measuring range of 0 to $100 \mathrm{cN}$ and three yarn guides on the measuring head, were also integrated into the yarn path between the roller and the needle. Two position adjustable ceramic yarn guides were also

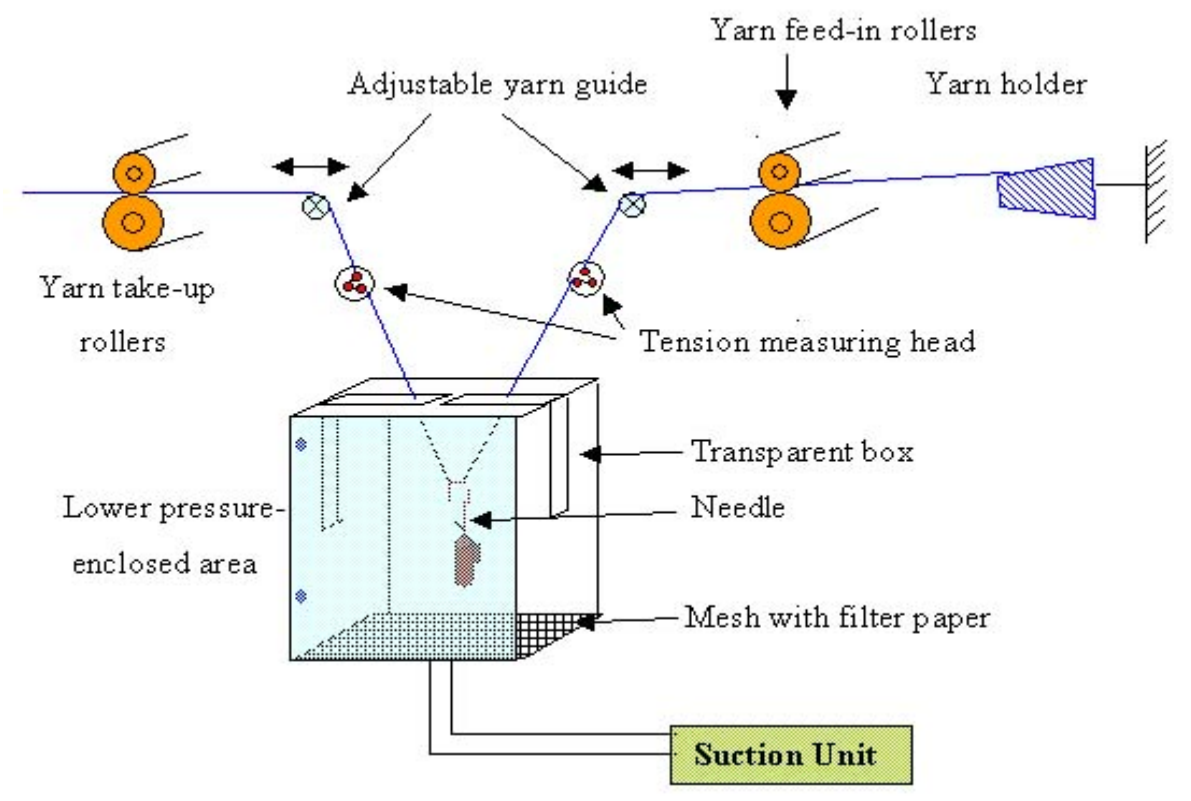

Fig. 3. Schematic diagram of the test rig. 
Table 1. Yarn specification

\begin{tabular}{cccc}
\hline Fiber Type & American cotton & Tenacity(cN/Tex) & 12.25 \\
\hline Yarn Count(tex) & 20 & Elongation at break(\%) & 4.05 \\
Yarn Twist(tpi) & 16.8 & Yarn Coefficient & 0.245 \\
Draft Ratio & 30.5 & Hairiness $>\mathbf{3 m m})$ & 3.88 \\
\hline
\end{tabular}

Table 2. Cotton yarn type for test

\begin{tabular}{cccc}
\hline Yarn Type & $\begin{array}{c}\text { Ring-Spun } \\
\text { Carded(RS) }\end{array}$ & $\begin{array}{c}\text { Ring-Spun } \\
\text { Combed(RC) }\end{array}$ & $\begin{array}{c}\text { Open-end } \\
\text { (OC) }\end{array}$ \\
\hline $\begin{array}{c}\text { Moisture } \\
\text { Content(\%) }\end{array}$ & $0 / 4 / 8$ & $0 / 4 / 8$ & $0 / 4 / 8$ \\
\hline
\end{tabular}

included in order to facilitate the change of yarn contact angle in the needle hook. The test yarn was threaded through the needle hook under tension, and the yarn movement was controlled with two pairs of rollers that were driven separately by two stepper motors.

During the test, the operating condition was maintained at $3 \mathrm{cN}$ of yarn tension, $45^{\circ}$ of yarn feeding angle, and $300 \mathrm{~m} / \mathrm{min}$. of the yarn feeding speed which were simulated to current knitting condition in the knitting process.

The main variables for the test were yarn types and moisture content of the yarn with respect to needle contamination. The yarn for the test was prepared with almost similar specification but different types and moisture condition as shown Table 1 and 2. Moisture content of yarn was adjusted with different wetting conditions in a standard condition. The test was carried 3 times in each testing condition.

The amount of fly shed during the test was determined by weighing the filter paper before and after testing. The fly accumulated on the filter paper was kept in a standard atmosphere room for 1 day before the measurement.

In addition, the total amount of fly was determined by measuring the weight of the yarn before and after testing at standard conditions using the following equations.

Where, wt\% : fly weight percent

$\mathrm{W}_{\mathrm{o}}$ : original yarn weight

$\mathrm{W}$ : weight of the amount of fly shed at the needle point

\section{Results and Discussion}

Fig. 4 showed the amount of contamination according to yarn types. Comparing combed yarn with carded in the ring-spun yarn, carded yarn had slightly higher amount of contamination than combed yarn. The combed ring-spun yarn has less short fibers such as lint than carded ringspun yarn due to the more purified spinning process. In addition, the combed ring-spun yarn had less contaminated materials from the harvesting such as soil and insect which may be removed from the spinning process than carded ring-spun yarn. However, open-end yarn had slightly higher amount of contamination than carded and combed ring-spun yarn. This result also showed that the open-end yarn may have much more short fibers which are not strongly adhered to the yarn body due to the shorter spinning process than ring spinning process.

It is also possible that the open end yarn has more contaminated materials in the yarn structure which come out during the knitting because of the friction between the yarn and needles. As results, it showed that the needle contamination in the knitting zone is also largely affected from the yarn types with different spinning method.

Fig. 5 showed needle contamination results according to yarn type and moisture contents.

The amount of contamination showed little but significant difference among the yarn types and moisture contents.

These results may be caused by more brittle yarns of low moisture contents which bring to severe frictional condition between needles and 


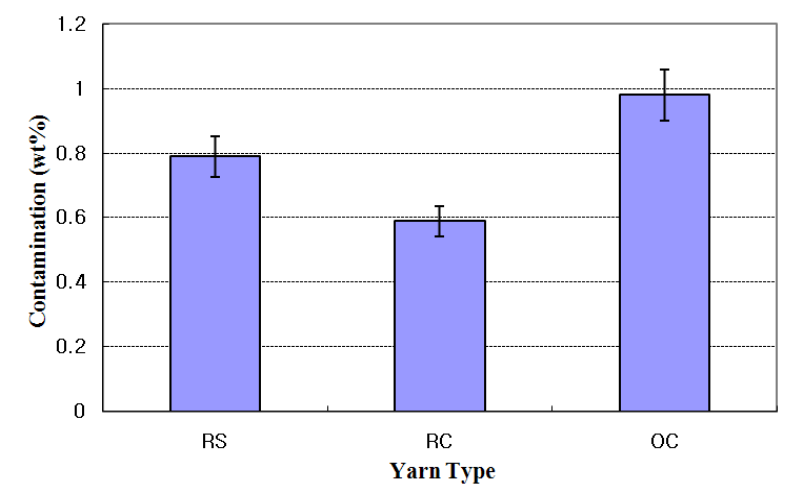

Fig. 4. The amount of contamination depending on different yarn types (moisture contents : 8\%).

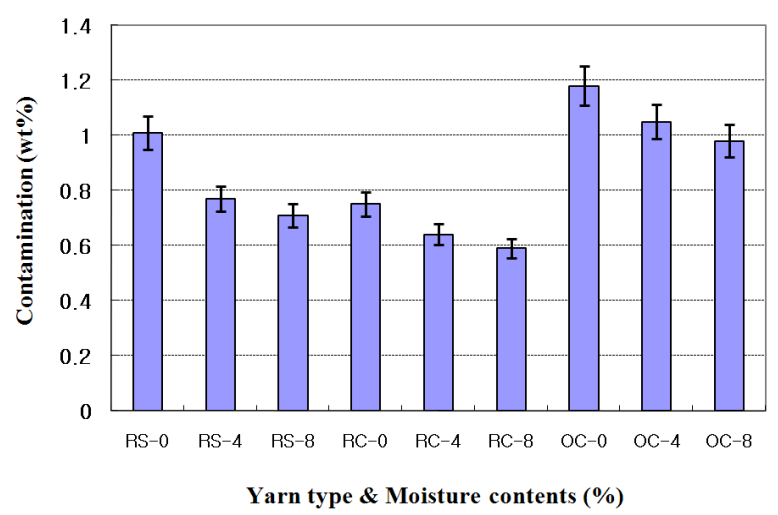

Fig. 5. The amount of contamination depending on different yarn types and moisture contents.

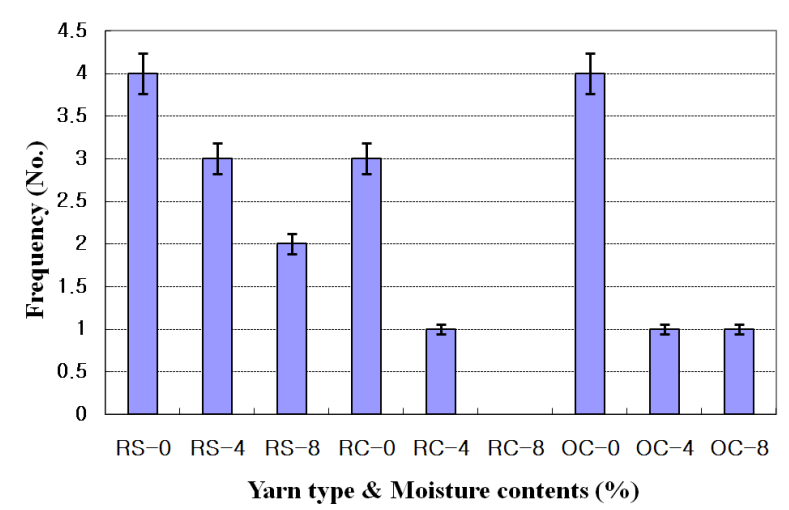

Fig. 6. Yarn breakage frequency depending on different yarn types and moisture contents (No. per 1 $\mathrm{km}$ length).

yarns then abstracting short fibers from yarn body.

As the knitting area experiences continuous frictional force between yarn and needle, it is strongly required to keep an optimum friction and tension among knitting elements especially with respect of needle contamination. Therefore, it is are very important to keep optimum moisture contents of yarn and moisture condition around

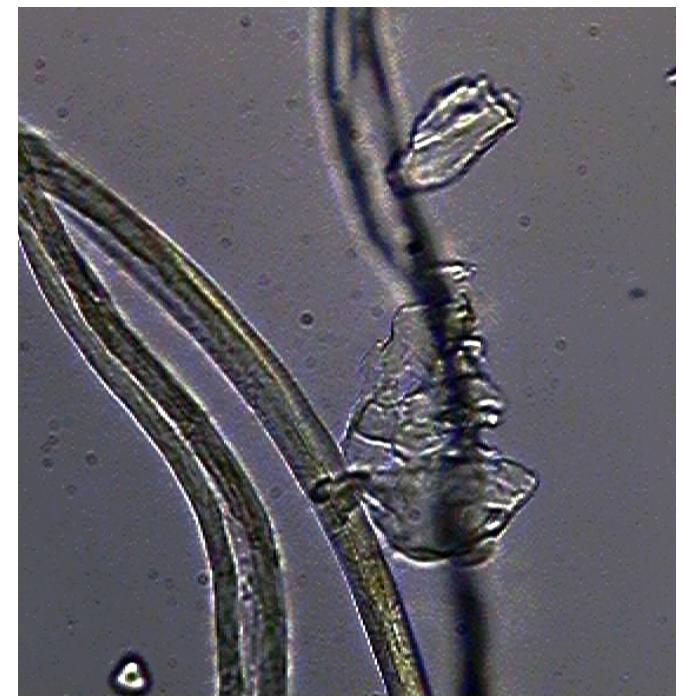

Fig. 7. Microscopic image of short fly and particles collected from the test.

knitting area in the knitting process.

Fig. 6 showed the frequency of yarn breakage during the test. Yarn breakage is possibly related to frictional force, yarn structure and moisture contents. Particularly, there may be a high frictional force on inside needle hook depending on the yarn feeding and yarn conditions. As shown from the image in Fig. 7, some particles which are collected during the test could be another potential reason to the yarn breakage and needle contamination. Therefore, highly controlled purification of yarn especially used for the knitting process is highly recommended for diminishing the problem of needle contamination during knitting.

\section{Conclusions}

Needle contamination is one of the serious problems in the knitting process which induces a delay of the process by cleaning, defacts of end-products, needle abrasion and wrong operation of needle. The needle contamination in knitting area is largely affected by yarn structure according to the yarn spinning process and it determines the quality of yarn with respect to staple length and impurities inside yarn structure.

Because it is difficult to define the extent of needle contamination directly from very com- 
plicated knitting zone in real knitting process, the needle contamination was investigated using a developed test rig which simulated the knitting zone.

The results showed that needle contamination was significantly influenced by the yarn structure particularly the condition of short fibers which is a main cause of the contamination.

Moisture content of the yarns was also critical to the contamination because low moisture content of yarn induces high friction between yarn and needle producing short fibers and impurities which may be detached from yarns.

Therefore, we concluded that it is highly recommended to choose more purified yarns with suitable moisture content for the knitting process.

\section{Acknowledgments}

This work was supported for two years by Pusan National University research grant.

\section{References}

1. G. Bühler, O. Riederer, W. Haussler, and G. Egbers, The Reduction of Fibre Fly by Obtaining the Best Possible Results from the Knitting Yarn, Part 1: the Effect of Fibres and Yarns, Knitting Technique, 12, 208-211(1990).

2. G.F. Ruppenicker, and J.T. Lofton, Factors Affecting the Lint Shedding of Cotton Knitting Yarns, Textile Res. J., 49, 681-685(1979).

3. C.A. Lawrence and S.A. Mohamed, Yarn and Knitting Parameters Affecting Fluff-during
Weft Knitting of Staple Yarns, Textile Res. J., 66, 694-704(1996).

4. Y.S. Koo, Correlation of Yarn Tension with Parameters in the Knitting Process, Fibers and Polymers, 3, 80-84(2002).

5. Y.S, Koo, Knitting Parameters on Lint Pollution during Knitting Process, Textile Coloration and Finishing(J. Korean Soc. Dyers \& Finishers), 18(3), 179-184(2006).

6. Y.S. Koo, Bending Behavior of Coated Yarns, Fibers and Polymers, 2, 149-152(2001).

7. W.R. Evans, Cotton Knitting 'Fly' Problems and some Solutions, Knitting International, May, pp.41-43, 1983.

8. BÜhler $\mathrm{G}$, Riederer $\mathrm{O}$, Haussler $\mathrm{W}$, Egbers G., The Reduction of Fibre Fly by Obtaining the best possible Results from the Knitting Yarn, Part1: The Effect of Fibres and Yarns, Knitting Technique, 12, 208-211(1990).

9. B. Edberg, "Cotton Dust Elimination in Weft Knitting”, Knitting Times Yearbook, U.K., pp.88-89, 1985.

10. D. E. Smith, N.D. Burns, and G.R.Wray, The Frictional Forces between Yarns and Weftknitting Elements, J. Text. Inst., 65, 337-345 (1974).

11. H. G. Jong, Yarn to Yarn Friction in relation to some Properties of Fibre Materials, Textile Res. J., 63, 14-18(1993).

12. Y.S. Koo, Yarn Hairness Affecting Fluff Generation, Fibers and Polymers, 4, 119-123 (2003). 